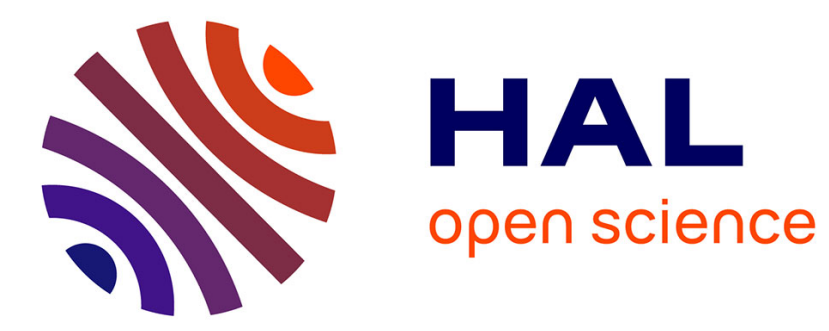

\title{
Traffic Grooming in Bidirectional WDM Ring Networks
}

\author{
Jean-Claude Bermond, David Coudert, Xavier Munoz, Ignasi Sau
}

\section{To cite this version:}

Jean-Claude Bermond, David Coudert, Xavier Munoz, Ignasi Sau. Traffic Grooming in Bidirectional WDM Ring Networks. International Conference on Transparent Optical Networks (ICTON), Jun 2006, Nottingham, United Kingdom. pp.19 - 22, 10.1109/ICTON.2006.248390 . inria-00429168

\section{HAL Id: inria-00429168 https://hal.inria.fr/inria-00429168}

Submitted on 1 Nov 2009

HAL is a multi-disciplinary open access archive for the deposit and dissemination of scientific research documents, whether they are published or not. The documents may come from teaching and research institutions in France or abroad, or from public or private research centers.
L'archive ouverte pluridisciplinaire HAL, est destinée au dépôt et à la diffusion de documents scientifiques de niveau recherche, publiés ou non, émanant des établissements d'enseignement et de recherche français ou étrangers, des laboratoires publics ou privés. 


\title{
Traffic Grooming in Bidirectional WDM Ring Networks \\ (extended abstract)
}

\author{
Jean-Claude Bermond ${ }^{1}$, David Coudert ${ }^{2}$, Xavier Muñoz ${ }^{3}$ and Ignasi Sau ${ }^{4}$ \\ ${ }^{1}$ Jean-Claude.Bermond@sophia.inria.fr, David.Coudert@sophia.inria.fr, ${ }^{3} x m l @ m a 4 . u p c . e d u$, \\ 4ignasi@ma4.upc.edu
}

\begin{abstract}
We study the minimization of ADMs (Add-Drop Multiplexers) in Optical WDM Networks with Bidirectional Ring topology considering symmetric shortest path routing and all-to-all unitary requests. We insist on the statement of the problem, which had not been clearly stated before in the bidirectional case. Optimal solutions had not been found up to date. In particular, we study the case $C=2$ and $C=3$ (giving either optimal constructions or near-optimal solutions) and the case $C=k(k+1) / 2$ (giving optimal decompositions for specific congruence classes of $N$ ). We state a general Lower Bound for all the values of $C$ and $N$, and we improve this Lower Bound for $C=2$ and $C=3$ (when $N=4 t+3$ ). We also include some comments about the simulation of the problem using Linear Programming.
\end{abstract}

Keywords: SONET over WDM; traffic grooming; ADM; MILP formulation; graph decomposition; combinatorial designs.

\section{1- INTRODUCTION AND STATEMENT OF THE PROBLEM}

Traffic grooming in networks refers to grouping low rate traffic into higher speed streams (see $[1,2,3]$ ). There are many variants according to the type of network considered (for example, in [4] the Path grooming problem is studied), the constraints used and the parameters to be optimize which give rise to a lot of interesting design problems (graph decomposition).

The objective is to minimize the equipment cost. Among possible criteria, one is to minimize the number of wavelengths used to route all the requests. This leads to the widely studied loading problem [5]. Another choice, which is in fact a better approximation of the real equipment cost, is to minimize the number of add/drop locations (called ADMs in SONET terminology) instead of the number of wavelengths. These two problems are proved to be different. Indeed, it is known that even for the simpler network (the unidirectional ring), the number of wavelengths and the number of ADMs cannot be simultaneously minimized [6]. In [7] the bidirectional case is studied, and in [8] a MILP formulation of the traffic grooming in the ring is done.

Let an optical network be represented by a directed graph $G$ (in many cases a symmetric one) on $N$ vertices, for example an unidirectional ring $C_{N}$ or a bidirectional ring $C_{N}{ }^{*}$. We are given also a traffic (or instance) matrix, that is a family of connection requests represented by a multidigraph $I$ (the number of arcs from $i$ to $j$ corresponding to the number of requests from $i$ to $j$ ). It is usual to refer to $G$ as the physical graph, whereas $I$ is called logical graph (or request graph). Satisfying a request $r$ from $i$ to $j$ consists in finding a route (path) $P(r)$ in $G$ and assigning a wavelength to it. The grooming factor (or grooming ratio) $C$ is the number of unitary requests that can be grouped on a single wavelength, i.e. a request uses only $1 / C$ of the bandwith available on a wavelength along its route. In other words, for each arc $e$ of $G$ and for each wavelength $w$, there are at most $C$ paths using wavelength $w$ and containing $\operatorname{arc} e$. Let $B_{w}$ be the subgraph of $I$ that represents the set of instances that use wavelength $w$. Then the load is defined as follows

Definition 1.1 For a subgraph $B_{w}$ of requests of $I$, we define the load of an edge $e$ of $G, L\left(B_{w}, e\right)$, as the number of requests which are routed through $e$. i.e.:

$$
L\left(B_{\omega}, e\right)=\left|\left\{P(r) ; r \in E\left(B_{\omega}\right) ; e \in P(r)\right\}\right|
$$

Work suported by COST action 293: Graphs and Algorithms in Communication Networks. Partially supported by the Spanish Research Council under Projects CICYT, TIC2000-1017 and TIC2001-2171 
For each wavelength $w$, an $\mathrm{ADM}$ is needed at each node sending or receiving a request on that wavelength. Recall that only one ADM is needed for a node to send or receive various requests on the same wavelength. Therefore The number of ADMs used on wavelength $w$ equals the number of vertices in the the subgraph $B_{w}$.

Let $A_{r}$ be the set of paths in $G$ through which a request $r$ may be routed and let $A=\bigcup_{r \in I} A_{r}$. The general

Traffic Grooming Problem is stated as follows:

Problem 1.1 (The Traffic Grooming Problem)

Input : $A$ digraph $G$ (network), a digraph I (set of requests), a set $A$ (allowed paths) and a grooming factor $C$

Output : Find for each arc $r \in I$ a path $P(r) \in A_{r}$, and a partition of the arcs of $I$ into subgraphs $B_{w}, 1 \leq w \leq W$, such that $\forall e \in E(G) L\left(B_{\omega}, e\right) \leq C$

Objective : Minimize $\sum_{w=1}^{W}\left|V\left(B_{w}\right)\right|$, and this minimum is denoted $A(G, I, A, C)$

In this work we focus on the Bidirectional Ring Grooming Problem with symmetric shortest path routing, and specifically the all-to-all unitary case. i.e., from now on we will consider $G=C_{N}$ and $I=$ $T_{N}$, where $T_{N}$ is a tournament containing all the $\operatorname{arcs}(i, i+q), i=0, \ldots, N-1, q=1, \ldots,\lfloor N / 2\rfloor$ (plus $N / 2$ arcs of the form $(i, i+N / 2)$, if $N$ is even).

Remark 1.1 In this case, what we do is minimize the number of ADMs used by the requests following one direction in the cycle, and then double the number of ADMs and the number of wavelengths to compute the total number of ADMs used by the whole set of requests. In this way, we can get rid of the orientation of the requests, because all of them have the same direction. This is the main reason for choosing this routing, besides of its common use in real optical networks. Thus, all the results that we will show take into account only half of the total number of ADMs.

Finally, our problem can be reformulated as follows.

\section{Problem 1.2 (Symmetric Shortest Path routing)}

Input : $\vec{C}_{N}$ : unidirectional cycle, and a grooming factor $C . A$ set of requests given by a tournament $T_{N}$. The request $(i, j)$ being routed by the shortest path, and if $(i, j)$ is routed in one direction, $(j, i)$ is routed in the opposite direction

Output : Find for each arc $r \in T_{N}$ a path $P(r)$ in $\vec{C}_{N}$, and a partition of the arcs of $K_{N}$ into subgraphs $B_{w}, 1 \leq w \leq W$, such that $\forall e \in E\left(\vec{C}_{N}\right) L\left(B_{\omega}, e\right) \leq C$

Objective : Minimize $\sum_{w=1}^{W}\left|V\left(B_{w}\right)\right|$, and this minimum is denoted $A(C, N)$

\section{2- GENERAL LOWER BOUNDS}

Let's introduce some notation: consider a valid construction for the Problem and let $a_{p}$ denote the number of subgraphs of the partition with exactly $p$ nodes, $A$ the number of ADMs, and $W$ the number of subgraphs of the partition.

We have the following equalities:

$$
\begin{aligned}
A & =\sum_{p=2}^{N} p a_{p} \\
\sum_{p=2}^{N} a_{p} & =W \\
\sum_{w=1}^{W}\left|E_{w}\right| & =|E|
\end{aligned}
$$

In the particular case where $I=T_{N}$, we have $|E|=N(N-1) / 2$, and we know that

$$
W \geq\left\lceil\frac{N^{2}-\varepsilon}{8 C}\right\rceil, \text { where } \varepsilon= \begin{cases}0, & \text { if } N \text { even } \\ 1, & \text { if } N \text { odd }\end{cases}
$$

To obtain accurate lower bounds we need to bound the value of $\left|E_{\omega}\right|$ for a graph with $\left|V_{\omega}\right|=p$ vertices, satisfying the load constraint. 
Definition 2.1 Let $\gamma(C, p)$ be the maximum number of edges of any graph $H$ with $p$ vertices, such that $L(H, e) \leq C, \forall e \in H$.

\section{Proposition 2.1 (Requests of Shortest Length)}

Let $C=k(k+1) / 2+r$, with $0 \leq r \leq k$, then $\gamma(C, p)$ is given by the expression

$$
\gamma(C, p)=\left\{\begin{array}{cl}
\frac{p(p-1)}{2} & , \text { if } p \leq 2 k+1+\varepsilon, \text { with } \varepsilon=1 \text { if } r \geq \frac{k+2}{2} \\
k p+\left\lfloor\frac{r p}{k+1}\right\rfloor & , \text { otherwise }
\end{array}\right.
$$

Using the previous result and equations (1), (2) and (3), we state a general Lower Bound for our Problem:

\section{Theorem 2.1 (General Lower Bound)}

For $G=\vec{C}_{N}, \mathrm{C}=k(k+1) 2+r$, with $0 \leq r \leq k$, and if all the requests cannot be put in a single subgraph:

$$
A(C, N) \geq\left\lceil\frac{N(N-1)}{2} \frac{k+1}{k(k+1)+r}\right\rceil
$$

It is easy to se that for $C=1$ the lower bound can always be reached using the decomposition in cycles that can be found in $[10]$.

\section{3- CASE C $=2$}

We have improved the previous Lower Bound when the grooming factor is equal to 2 .

\section{Proposition 3.1 (Tighter Lower Bound for $\mathrm{C}=2$ )}

For $G=\vec{C}_{N}$ and $\mathrm{C}=2$,

$$
A(2, N) \geq\left\lceil\frac{11 N(N-1)}{32}\right\rceil
$$

To get an idea about the validity of this Lower Bound, we have formulated the Problem using MILP, taking into account equations (1), (2) and (3). A graph of the ratio $A D M_{-}$simplex/ADM_LB vs $L o g(N)$ is drawn in Fig. 1 , for $N>14$. We can observe in this graph that the ratio tends to 1 when $N \overline{\text { increases. Notice }}$ that a solution given by MILP does not imply the existence of a valid decomposition, since the load of edges is not taken into account.

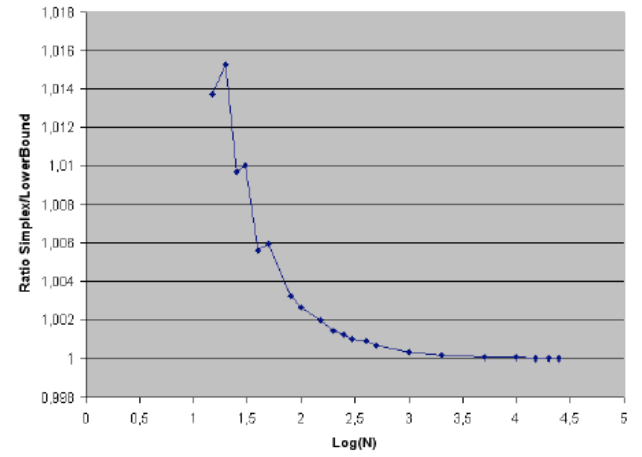

Figure 1. Simplex/LB vs $\log (N)$

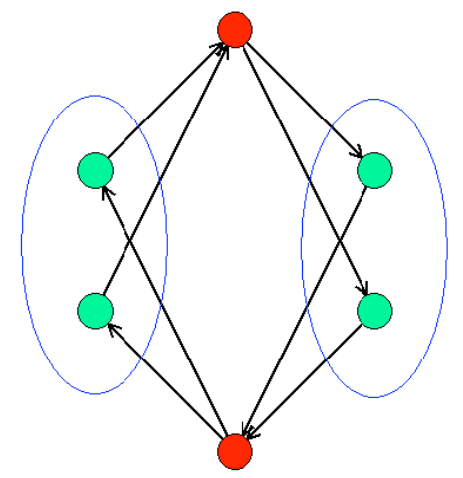

Figure 2. Graph used in the 12/11-approx

We have found explicit decompositions which are near-optimal solutions for $C=2$. In particular, we proved that for odd values of $N, T_{N}$ can be decomposed into triangles and subgraphs isomorphic to the one depicted in Fig. 2 in such a way that the load of every edge is less or equal to 2 and the number of ADMs given by such decomposition is less than 12/11 times the lower bound.

\section{4- CASE C $=3$}

Similarly to the previous case, we have improved the Lower Bound when $N=4 t+3$, and we have found either optimal solutions (see Section 6) or 1-approxs (extending the Steiner triples construction in [11]) 
for all the odd values of $N$, as we can see in Table 1 . The extension to the even values of $N$ should be straightforward.

\begin{tabular}{|c|c|c|}
\hline$N \quad(\bmod 12)$ & Lower Bound & ADMs of the explicit construction \\
\hline \hline 1,5 & $\frac{N(N-1)}{4}$ & $\frac{N(N-1)}{4}$ \\
\hline 3,7 & $\frac{N(3 N-1)}{12}$ & $\frac{N(3 N-1)}{12}+\frac{N}{3}-3$ \\
\hline 9 & $\frac{N(N-1)}{4}$ & $\frac{N(N-1)}{4}+4$ \\
\hline 11 & $\frac{N(3 N-1)}{12}$ & $\frac{N(3 N-1)}{12}+\frac{N}{3}-11$ \\
\hline
\end{tabular}

Table 1. Explicit constructions for $C=3$ and $N$ odd

\section{5- CASE C $=k(k+1) / 2$}

Using the results of the unidirectional case, we have found optimal constructions for several values of $C$ and infinite families of values of $N$. The main idea is to take known decompositions of the unidirectional case (using combinatorial designs that are in fact BIBDs, see [1] and [11]), and then split each vertex (except one) to obtain graphs that become optimal for the bidirectional case (under criterion of Proposition 2.1). In Table 2 we sum up the values for which we have obtained optimal solutions.

\begin{tabular}{|c|cc|}
\hline$C$ & \multicolumn{2}{|c|}{$N$} \\
\hline 3 & $N \equiv 1,5$ & $(\bmod 12)$ \\
6 & $N \equiv 1,7$ & $(\bmod 24)$ \\
10 & $N \equiv 1,9$ & $(\bmod 40)$ \\
\hline
\end{tabular}

\section{6- CONCLUSIONS}

Table 2. Optimal solutions

In this paper we have formally stated the Traffic Grooming Problem considering symmetric shortest path routing and all-to-all unitary requests, and we have given a general Lower Bound. It would be interesting to try to improve this LB for other values of $C$ (besides 2 and 3), but it seems to be a difficult problem.

Finding other optimal constructions will lead to exciting graph decomposition problems, where tools from combinatorial design are strongly needed. Another approach to the problem consists in finding good approximations, which sometimes (but not always) has more practical value.

Other routings and topologies could be considered, and we are convinced that many of the results that we sum up in this paper can be extended to similar problems.

\section{REFERENCES}

J.-C. Bermond, D. Coudert and X. Muñoz: Traffic grooming in Unidirectional WDM Ring Networks: the all-to-all unitary case. ONDM 03, 7th IFIP Working Conference on Optical Network Design and Modelling 2003, pp 1135 - 1153, 3-5 February.

J.-C. Bermond, et al: Traffic grooming in unidirectional WDM rings with grooming ratio $\mathrm{C}=6$. SIAM Journal on Discrete Mathematics, 19 (2005), pp. 523 - 542.

J.-C. Bermond and D. Coudert: Traffic Grooming in unidirectional WDM ring networks using design theory. IEEE ICC 2003, ON07-3.

J.-C. Bermond, L. Braud and D. Coudert: Traffic Grooming on the Path. SIROCCO Vol. 3499 of LNCS, (2005), pp. 34 - 38.

B. Beauquier, et al: Graph problems arising from wavelength-routing in all-optical networks. In IEEE Workshop on Optics and Computer Science, Geneva, 1997

A. Chiu and E.H. Modiano: Traffic grooming algorithms for reducing electronic multiplexing costs in WDM ring networks. IEEE/OSA Journal of Lightwave Technology, 2000, 18(1) :2-12

Charles J. Colbourn and Peng-Jun Wan: Minimizing Drop Cost for SONET/WDM Networks with 1/8 Wavelength Requirements. Networks Vol. 37(2), 107-116 2001.

J. Hu: Traffic Grooming in WDM Ring Networks: A Linear Programming Solution, OSA Journal of Optical Networks 1(11) 2002, pp. 397 - 408

J.-C. Bermond, L. Chacon, D. Coudert, and F. Tillerot: Cycle Covering. International Colloquium on Structural Information and Communication Complexity -- SIROCCO, Proceedings in Informatics 11, Vall de Nuria, Spain, pages 21-34, 27-29, June 2001

Charles J. Colbourn and Jeffrey H. Dinitz: The CRC Handbook of Combinatorial Designs. 1996 by CRC Press, Inc 